\title{
L'importanza della safety delle terapie cardiovascolari: aspetti di metodo e applicazioni
}

\author{
Achille P. Caputi $^{(1)}$, Gianluca Trifirò ${ }^{(1,2)}$
}

\begin{abstract}
I farmaci cardiovascolari rappresentano tradizionalmente una grossa fetta della spesa farmaceutica in Italia così come negli altri Paesi occidentali. Il rapporto dell'Osservatorio Nazionale sull'Impiego dei Medicinali (OsMed) per il periodo gennaio-settembre 2009 ha riportato che i farmaci cardiovascolari rappresentano la principale categoria terapeutica nel nostro Paese, sia in termini di Dose Definite Die per 1.000 abitanti die (48\% del totale) che di spesa pro capite $(37 \%)$ [1].

L'impiego di farmaci cardiovascolari nella pratica clinica è generalmente cronico e si inserisce nel contesto di politerapie, nel corso delle quali la safety rappresenta un aspetto rilevante. Infatti, durante l'esposizione cronica ai farmaci cardiovascolari possono insorgere occasionalmente reazioni avverse fatali o che richiedono ospedalizzazione, con conseguente impatto su morbidità e mortalità. Basti pensare al celebre caso di cerivastatina, farmaco ipocolesterolemizzante che è stato ritirato dal mercato dalla casa produttrice (Bayer) nell'agosto 2001, a causa di un aumentato rischio di rabdomiolisi. Dopo il ritiro di cerivastatina, si è sviluppata una accesa polemica sul potenziale conflitto di interesse da parte della agenzia regolatoria americana Food and Drug Administration in merito al ritiro tardivo dal mercato di tale farmaco. Infatti, alcune analisi retrospettive delle banche dati di segnalazione spontanea di reazione avversa avevano mostrato che, già a partire dal dicembre 1999, cerivastatina in monoterapia risultava associata a un aumento di dieci volte del rischio di rabdomiolisi rispetto alle altre statine [2].
\end{abstract}

Al di là dell'impatto diretto della safety delle terapie cardiovascolari e delle difficoltà nella sua corretta valutazione, l'insorgenza di reazioni avverse rappresenta una delle principali motivazioni per la sospensione e la ridotta aderenza a terapie croniche con farmaci, quali statine o antipertensivi, influenzando in tal modo anche l'efficacia di questi farmaci. Uno studio americano ha osservato che circa il $20 \%$ delle terapie croniche con farmaci ipolipemizzanti viene interrotto a causa di insorgenza di eventi avversi in pratica clinica, percentuali significativamente superiori rispetto a quelle riportate nei trial clinici randomizzati (RCT) [3].

Alla luce delle evidenze sopra riportate, appare evidente che sia necessario un costante e attento monitoraggio post-marketing della sicurezza a breve e lungo termine delle terapie cardiovascolari.

Nel corso di tale articolo saranno passate in rassegna le maggiori criticità relative alla valutazione pre- e post-marketing della sicurezza delle terapie cardiovascolari.

\section{TRIAL CLINICI: QUALE RUOLO NELLA VALUTAZIONE DELLA SAFETY?}

Nella comunità scientifica sono da tempo noti i limiti degli RCT nella valutazione della safety dei farmaci. Il campione selezionato e numericamente ridotto dei pazienti arruolati, la limitata durata del follow up, gli endpoint clinici predefiniti, l'attento monitoraggio da parte dei medici rendono da un lato gli RCT un perfetto scenario sperimentale per testare l'efficacia del farmaco ma, dall'altro, non soddisfano i criteri per una corretta valutazione della sicurezza a breve e, soprattutto, a lungo termine delle terapie farmacologiche.

Inoltre, i risultati degli RCT non possono essere direttamente trasferiti alla pratica clinica, in cui le condizioni di impiego dei farmaci sono notevolmente differenti. In particolare, l'uso cronico così come la politerapia sono aspetti fondamentali delle terapie cardiovascolari che non possono essere adeguatamente presi in considerazione nel corso degli RCT pre-marketing.

Numerosi studi hanno recentemente confrontato le caratteristiche dei pazienti con patologia cardiovascolare arruolati negli RCT rispetto a quelli affetti da tali patologie nella pratica clinica. Alcuni Autori americani hanno osservato che solo il $25 \%$ e il $9 \%$ di 593 recenti RCT di pazienti con sindrome coronarica acuta avevano rispettivamente arruolato donne e pazienti con più di 75 anni. Gli Autori hanno concluso che la sottorappresentazione di questi pazienti negli RCT limita la possibilità di fornire cure basate su evidenze a tutti i pazienti con patologia cardiovascolare [4].
${ }^{(I)}$ Dipartimento di Medicina Sperimentale e Farmacologia, Università di Messina (2) Erasmus University Medical Center, Rotterdam 
Indipendentemente dai limiti intrinseci agli RCT, il caso rofecoxib, ritirato tardivamente dal mercato a causa di un aumento del rischio di gravi eventi avversi cardiovascolari nel settembre 2004, ha spinto le agenzie regolatorie internazionali e la comunità scientifica ad interrogarsi su quali azioni intraprendere per migliorare la valutazione pre-marketing della safety dei farmaci.

È stata specificamente posta l'attenzione sulla possibilità di migliorare le procedure di approvazione al commercio dei farmaci, basate sui risultati degli RCT pre-marketing, al fine di immettere sul mercato principi attivi il più possibile efficaci e sicuri.

Nello specifico, era stata avanzata l'ipotesi di richiedere evidenze statistiche maggiori (cut-off per il p-value $<0,0001$ invece che il tradizionalmente accettato $<0,05$ ) sull'efficacia dei farmaci negli RCT pre-marketing, così da rallentare il processo di approvazione del farmaco, garantendo automaticamente una migliore valutazione della safety. Tuttavia, tale ipotesi è stata fermamente rigettata da esperti autorevoli, come riportato in alcuni editoriali pubblicati su Circulation $[5,6]$. Tali autori hanno sottolineato che è comunque indispensabile un attento monitoraggio post-marketing per una accurata valutazione della safety dei farmaci e hanno inoltre aggiunto che il rallentamento del processo di immissione in commercio di nuovi principi attivi potrebbe privare temporaneamente i medici di valide opzioni terapeutiche.

\section{STUDI OSSERVAZIONALI: LIMITI E POTENZIALITÀ NELLA VALUTAZIONE DEL RISCHIO}

Nell'ultimo decennio, la crescente disponibilità di banche dati sanitarie negli USA e in Europa ha favorito la diffusione di studi osservazionali post-marketing sulla safety sia a breve sia a lungo termine delle terapie farmacologiche, incluse quelle cardiovascolari. Esistono dei limiti di questi studi che devono però essere attentamente presi in considerazione.

A differenza dei trial clinici, nelle banche dati sanitarie i dati sono registrati sistematicamente a fini burocratici-amministrativi ed economici (es. rimborso di prescrizioni di farmaci o dimissioni ospedaliere) e non di ricerca. Da una parte, questa caratteristica previene il cosidetto selection bias, in quanto tutti i pazienti registrati nella banca dati sono potenzialmente arruolabili nello studio, evitando qualunque tipo di selezione sulla base di criteri di inclusione che possano influenzare i risultati dello studio. Dall'altra parte, queste banche dati non garantiscono lo stesso livello di precisione e di completezza degli RCT riguardo le informazioni cliniche dei pazienti.

Un altro limite principale degli studi osservazionali rispetto agli RCT è l'assenza di ran- domizzazione nell'assegnazione dei pazienti ai diversi gruppi di trattamento. Nella pratica clinica, infatti, diversi fattori guidano il medico prescrittore nella scelta di un farmaco, fenomeno che in farmacoepidemiologia viene definito channelling. Tuttavia, nuove tecniche promettenti, quali il multidimensional propensity score adjustment, permettono, almeno in parte, di riprodurre negli studi osservazionali uno scenario simile a quello sperimentale degli RCT.

Un altro aspetto metodologico rilevante da considerare negli studi osservazionali riguarda il confounding by indication. Con tale termine si indica il potenziale effetto di confondimento esercitato dall'indicazione d'uso di un farmaco sull'associazione tra esposizione al farmaco stesso e insorgenza di un evento avverso, che si verifica quando l'indicazione d'uso del farmaco è un fattore di rischio per l'evento studiato. Tale problema è emerso qualche anno fa nel corso di una delle controversie farmacoepidemiologiche sui possibili rischi associati all'uso dei calcio-antagonisti per il trattamento dell'ipertensione [7].

Alcuni studi osservazionali avevano osservato un'associazione tra calcio-antagonisti e rischio di infarto del miocardio e mortalità cardiovascolare. In altri studi però tale associazione veniva attribuita all'effetto del confounding by indication, in quanto l'ipertensione è sia un'indicazione d'uso per i calcio-antagonisti sia un fattore di rischio per eventi cardiovascolari. In aggiunta, un'analisi di Huse e colleghi [8] sull'uso dei farmaci antipertensivi, condotta tramite interviste a medici americani, aveva dimostrato che i calcio-antagonisti sono considerati dalla classe medica come farmaci particolarmente efficaci nel trattamento dei pazienti a più alto rischio cardiovascolare, piuttosto che di quelli con ipertensione lieve, avvalorando così l'ipotesi precedentemente postulata sul ruolo del confounding by indication.

Per eliminare l'effetto del confounding by indication esistono tuttavia delle valide strategie che possono essere messe in atto sia nella fase di disegno dello studio (es. restrizione del campione in studio a pazienti trattati per la stessa patologia, matching di casi e controlli con la stessa patologia) sia nel corso delle fasi di analisi dello studio (es. stratificazione, analisi multivariate).

Nonostante i limiti relativi all'uso delle banche dati, nel corso degli ultimi anni stanno emergendo altre potenziali applicazioni nell'ambito della valutazione della safety dei farmaci. Sono in corso alcuni progetti internazionali, quali EU-ADR (www.euadr-project.org) e FDA's Sentinel Initiative (http://www.fda.gov/Safety/ FDAsSentinelInitiative), che hanno l'obiettivo di esplorare sistematicamente $i$ dati di molteplici banche dati amministrative e di medicina generale al fine di identificare precocemente eventuali segnali di sicurezza associati a terapie 
farmacologiche. L'aggregazione dei dati provenienti da diverse banche dati, resa possibile da un complesso processo di armonizzazione, porta ad un vantaggioso incremento delle dimensioni e dell'eterogeneità della popolazione in studio; in tal modo, è possibile una precisa valutazione dei rischi associati a singoli farmaci, anche raramente utilizzati, e in specifici gruppi di pazienti, generalmente sottorappresentati negli RCT.

Tali approcci innovativi potrebbero supportare i tradizionali sistemi di segnalazione spontanea di sospette reazioni avverse nell'identificazione precoce dei segnali di safety e in più rappresentare validi strumenti per la valutazione della safety a lungo termine di terapie croniche, come quelle cardiovascolari.

In questo contesto, la Comunità Europea ha recentemente finanziato, all'interno del settimo programma quadro (FP7), diversi progetti (SOS - Safety of NSAIDs - www.sos-nsaids-project. org; ARITMO - www.aritmo-project.org) volti a valutare la safety cardiovascolare dei farmaci attraverso la combinazione di diverse banche dati, tra cui alcune italiane.

\section{LE INTERAZIONI FARMACOLOGICHE NEL CORSO DELLE TERAPIE CARDIOVASCOLARI: UN PROBLEMA APERTO}

Le interazioni farmacologiche giocano un ruolo importante nella safety delle terapie cardiovascolari. Per dare un'idea sulla dimensione di tale problema si riportano di seguito alcuni esempi. Nel febbraio 2004 1'Adverse Drug Reactions Bulletin ha riportato che l'uso concomitante di farmaci inibitori dell'isoenzima epatico CYP3A4 è il principale fattore di rischio di miotossicità da statine [9]. Inoltre, una review appena pubblicata sul management dell'ipertensione indica che uno dei fattori che possono peggiorare il controllo della pressione arteriosa è rappresentato dall'uso concomitante di sostanze o farmaci che possono alterare la pressione arteriosa o interagire con la terapia antipertensiva [10]. Ancora, per quanto riguarda warfarin, anticoagulante orale frequentemente utilizzato nella terapia di prevenzione cardiovascolare, esiste ad oggi un'evidenza consolidata sul rischio di interazione con più di 120 farmaci e fattori dietetici [11].

I limiti degli RCT nello studio delle interazioni farmacologiche e del loro possibile impatto sulla safety sono ancora più evidenti. I pazienti inclusi nei trial clinici sono quasi sempre trattati in monoterapia; soltanto alcune specifiche interazioni farmaco-farmaco sono analizzate tramite studi di farmacocinetica premarketing, ma raramente è valutato il loro potenziale impatto su endpoint primari.

Gli studi osservazionali sulle banche dati, invece, offrono la possibilità di valutare i rischi associati a interazioni farmacologiche, rappre- sentando uno strumento particolarmente utile per la definizione del profilo di rischio delle terapie cardiovascolari che sono assunte cronicamente e in schemi politerapici. A tal proposito, è in atto un acceso dibattito sugli effetti dell'interazione tra inibitori di pompa protonica e clopidogrel, antiaggregante che è frequentemente prescritto in associazione all'aspirina per la prevenzione cardiovascolare. Le linee guida internazionali raccomandano l'uso degli inibitori di pompa protonica, come gastroprotettori, nei pazienti che sono trattati con clopidogrel e aspirina. La sicurezza di tale interazione è stata però messa recentemente in discussione da tre grandi studi osservazionali condotti su banche dati che hanno osservato un piccolo, ma statisticamente significativo aumento di rischio di eventi cardiovascolare (rischio relativo compreso tra 1,25 e 1,5; comparatore: clopidogrel in monoterapia) [12]. Tuttavia, alcuni ricercatori hanno obiettato che i risultati di questi studi osservazionali potrebbero essere spiegati da un effetto del confounding by indication (es. uso maggiore di inibitori di pompa protonica in pazienti a più alto rischio cardiovascolare). Questo specifico caso mette ancora una volta in risalto le potenzialità e i limiti degli studi osservazionali su banche dati anche per quanto riguarda la valutazione del rischio associato alle interazioni farmacologiche.

Al fine di valutare quanto i medici siano consapevoli del rischio di interazione farmacologica in pazienti in terapia cronica con statine, uno studio di popolazione italiano ha analizzato qualche anno fa la co-prescrizione di statine e macrolidi a rischio di interazione, utilizzando $i$ dati della banca dati di medicina generale della Azienda Sanitaria Locale di Caserta [13].

Tale studio nasceva dalla considerazione che, a parte pravastatina, tutte le statine sono metabolizzate attraverso il sistema del citocromo P450 a livello epatico: l'isoenzima CYP3A4 metabolizza atorvastatina e simvastatina, mentre il CYP2C9 metabolizza fluvastatina e, in misura minore, rosuvastatina, substrato anche del CYP2C19 [14]. I macrolidi, antibiotici frequentemente prescritti in pratica clinica, possono aumentare la concentrazione plasmatica di alcune statine tramite l'inibizione dell'isoenzima CYP3A4, esponendo i pazienti al rischio di eventi avversi. Tuttavia, i macrolidi differiscono significativamente nel grado di inibizione del CYP3A4: eritromicina e claritromicina sono forti inibitori, mentre azitromicina e spiramicina hanno uno scarso potenziale di interazione $\mathrm{e}$, pertanto, andrebbero prescritti in pazienti in trattamento cronico con simvastatina e atorvastatina. In maniera sorprendente, il suddetto studio ha mostrato che gran parte dei medici di medicina generale co-prescrivevano statine e macrolidi ad alto rischio di interazione, nonostante avessero valide alternative terapeutiche, scevre da rischi di interazione farmacologica. 


\section{CONCLUSIONI}

Parafrasando Jerry Avorn, professore di Medicina alla Harvard Medical School di Boston, la valutazione della sicurezza di un farmaco segue le stesse fasi della valutazione clinica di un paziente [5]. Entrambi sono processi bayesiani in cui si cercano dei segnali, sulla base di un set di prior probabilities. Gli indizi di potenziali segnali devono essere successivamente esplorati, anche se non presentano inizialmente una chiara conferma di "significatività" del segnale. Infine, le ipotesi avanzate devono essere verificate tramite studi di hypothesis testing.

Le differenti fasi di questo complesso processo di valutazione della safety dei farmaci non possono essere simultaneamente ed esclusivamente prese in considerazione nel corso del processo di valutazione pre-marketing del farmaco. Tale assunzione è ancora più calzante per le terapie cardiovascolari che, essendo croniche e in politerapia, necessitano di una rivalutazione post-marketing della safety dopo diversi anni dalla loro immissione in commercio.

In attesa che le agenzie regolatorie internazionali migliorino le procedure di valutazione pre-marketing dei farmaci, vanno ulteriormente esplorate le potenzialità della banche dati sanitarie nel processo di valutazione del profilo rischio-beneficio delle terapie cardiovascolari nella pratica clinica. Nuovi approcci metodologici che sono stati intrapresi nell'ambito di diversi progetti internazionali supportano l'idea che le banche dati sanitarie siano delle fonti dati adatte sia per la conduzione di studi di hypothesis testing che per quelli di hypothesis generation, volti questi ultimi all'identificazione precoce di segnali di safety. In teoria, tali sistemi potrebbero portare al ritiro tempestivo di farmaci di cui si evidenzino gravi rischi, con l'obiettivo ultimo di prevenire casi simili a quelli di cerivastatina e rofecoxib.

\section{BIBLIOGRAFIA}

1. Agenzia Italiana del farmaco, Roma. L'uso dei farmaci in Italia - Rapporto gennaio-settembre 2009. Disponibile online su: http://www.agenziafarmaco.it/it/content//\%E2\%80\%99uso-dei-farmaci-italia-rapporto-gennaio-settembre-2009 (ultimo accesso: marzo 2011)

2. Psaty BM, Furberg CD, Ray WA, Weiss NS. Potential for conflict of interest in the evaluation of suspected adverse drug reactions: use of cerivastatin and risk of rhabdomyolysis. JAMA 2004; 292: 2622-31

3. Andrade SE, Walker AM, Gottlieb LK, Hollenberg NK, Testa MA, Saperia GM et al. Discontinuation of antihyperlipidemic drugs-do rates reported in clinical trials reflect rates in primary care settings? N Engl J Med 1995; 332: $1125-31$

4. Lee PY, Alexander KP, Hammill BG, Pasquali SK, Peterson ED. Representation of elderly persons and women in published randomized trials of acute coronary syndromes. JAMA 2001; 286: 708-13

5. Avorn J. Evaluating drug effects in the post-Vioxx world: there must be a better way. Circulation 2006; 113: 21736

6. Roth-Cline MD. Clinical trials in the wake of Vioxx: requiring statistically extreme evidence of benefit to ensure the safety of new drugs. Circulation 2006; 113: 2253-9

7. Joffe MM. Confounding by indication: the case of calcium channel blockers. Pharmacoepidemiol Drug Saf 2000; 9: $37-41$

8. Huse DM, Roht LH, Hartz SC. Selective use of calcium channel blockers to treat high-risk hypertensive patients. Pharmacoepidemiol Drug Saf 2000; 9: 1-9

9. Adverse Drug Reactions Advisory Committee (ADRAC). Australian Adverse Drug Reactions Bulletin 2004; 23 (1). Disponibile online su: http://www.tga.gov.au/adr/aadrb/aadr0402.htm (ultimo accesso: marzo 2011)

10. Basile JN, Bloch MJ. Identifying and managing factors that interfere with or worsen blood pressure control. Postgrad Med 2010; 122: 35-48

11. Hollbrook AM, Pereira JA, Labiris R, McDonald H, Douketis JD, Crowther M et al. Systematic overview of warfarin and its drug and food interactions. Arch Int Med 2005; 165: 1095-1106

12. Laine L, Hennekens C. Proton pump inhibitor and clopidogrel interaction: fact or fiction? Am J Gastroenterol 2010; 105: $34-41$

13. Piacentini N, Trifiró G, Tari M, Moretti S, Arcoraci V; UVEC group. Statin-macrolide interaction risk: a populationbased study throughout a general practice database. Eur J Clin Pharmacol 2005; 61: 615-20

14. Paoletti R, Corsini A, Bellosta S. Pharmacological interactions of statins. Atheroscler 2002; 3: 35-40 